\title{
THE ROLE OF HYBRID MODELS IN UNDERSTANDING FAILURE MECHANISMS OF INFRASTRUCTURE ELECTRONICS DUE TO HIGH-POWER MICROWAVE ILLUMINATION
}

\author{
D. C. Stoudt ${ }^{1}$ \\ R. L. Gardner ${ }^{2} \quad$ I Kohlberg $^{3}$
}

\begin{abstract}
Over the last few years, members of the electromagnetics community have used two types of models to investigate the differences in upset thresholds for multiple microsecond pulses compared to single or few shot short-pulse illuminations of complex electronic equipment. These models are: communication theory models and hybrid models. After reviewing and contrasting these models, we use a hybrid model to better understand failure modes in a SCADA trainer as a simple example. The model considers the type of failures seen in current injection testing of this SCADA. The purpose of the model is to introduce a methodology of predicting failure modes for complex infrastructure components.
\end{abstract}

\section{INTRODUCTION}

High-power microwave (HPM) tests have shown that extended illumination of some complex targets has shown lower thresholds than short-pulse illumination $[1,2]$. The extended illumination consists of hundreds of microsecond or longer pulses. The short-pulse illuminations are single shot or few pulse illuminations of around 100 nanoseconds duration. To understand why these electronic systems behave in this way various researchers have applied different types of models to appropriate types of electronic equipment.

Communications theory models determine the bit error rate between two components of a digital system and predict failure based on how that loss of information affects the function of the system. Hybrid models are functional models of systems that combine digital and analog systems or systems that have multiple discrete functional modes.

A number of researchers [3-6] have used communications theory to examine general digital systems. The digital system is modeled as a set of interconnected devices that store and process data and send the results along to the next processing unit. The communications between the two units can be by wires as in a computer bus or by other means. This problem is complicated by the potential of error detection and error-correction techniques in the communications link. Kohlberg and Gardner [7] applied this technique to early design (386 era) personal computers.

Hybrid Models [8] predict the behavior of systems with multiple states. A car with a manual transmission would be an example of such a system. In each gear, the car has a certain characteristic RPM versus speed curve. If the transmission is shifted into the lowest gear at the highest speed failure can occur. If the gear shift occurs too quickly then failure also is likely. Hybrid system failures often occur when there is an unwanted or abrupt transition between discrete states.

This paper will describe the basics of the two models and then use the hybrid model to describe a failure of a SCADA system. The prediction is compared with the behavior observed in a test facility used to test SCADAs and other related equipment against electromagnetic insult. The electronic equipment is installed in buildings as part of the test facility to make the tests as realistic as possible.

\section{ANALYTICAL MODELS}

There are two models that are used to predict particular types of upset for the equipment under test.

\subsection{Communications Theory Approach.}

When using this technique, models of the form shown in Fig. 1 are used. For a bus-type architecture the bus acts both as a good antenna and a data path. That is, various electronic components communicate with each other. Failure occurs when the system cannot recover from the data loss from the interference with the data communication.

\footnotetext{
${ }^{1}$ US Navy Distinguished Engineer for Directed Energy, Naval Surface Warfare Center, Dahlgren Division, Q04, 18444 Frontage Rd, Suite 324, Dahlgren, VA 22448-5161 USA.

Email: DLGR_NSWC_Q04_DE@navy.mil

${ }^{2}$ Consultant, Naval Surface Warfare Center, Dahlgren Division, Q04, 18444 Frontage Rd, Suite 324, Dahlgren, VA 22448-5161 USA Email: DLGR NSWC Q04 DE@navy.mil

${ }^{3}$ Kohlberg Assoc., Inc., PO Box 3525 Reston, VA 20195, USA.

Email: Ira.Kohlberg@gmail.com
} 


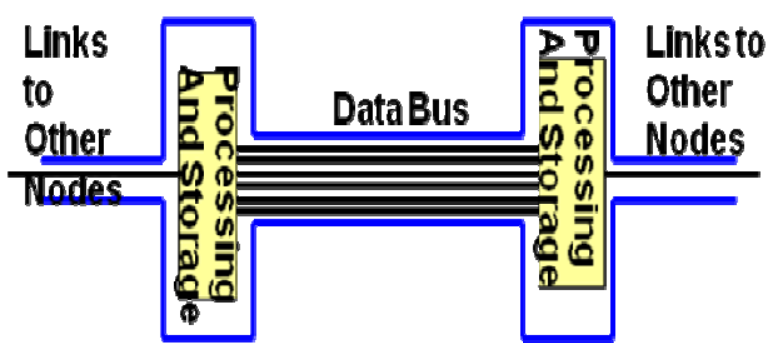

Figure 1: Simple bus architecture

Electromagnetic waves induce currents on the various antennas in the computer, such as the bus, and interfere with the signals on the bus. Interference levels near the signal levels are sufficient to cause bit errors. Some computer systems use error correction but most do not. The simplest form is for the computer to require retransmission of a data block when errors are detected. Kohlberg, Gardner and Ropiak [7] show that repeated retransmission causes such a system to halt. Note that the interference must cause repeated bit errors for an extended time to cause the upset so hundreds of threat pulses may be required. The pulses must also be of sufficient duration to be recognized as bits by the system.

\subsection{Hybrid Systems}

Hybrid systems combine digital and analog systems. Equation 1 describes a simple hybrid system.

$$
\dot{x}=\left(\begin{array}{cc}
0.1 & -1 \\
1 & 0.1
\end{array}\right) x+\left(\begin{array}{l}
1 \\
0
\end{array}\right) u
$$

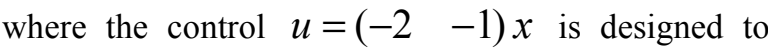
stabilize the system. In this example, the control will be applied $15 \mathrm{~s}$ after the system start. This system is, of course, unstable, but many systems of interest are unstable with control systems added to insure stability. Fig. 2 shows the evolution of this system. Sampling rate for the observer is critical for this system. If the sampling rate is sufficiently frequent, as shown, the system will stabilize. If some of the observations and

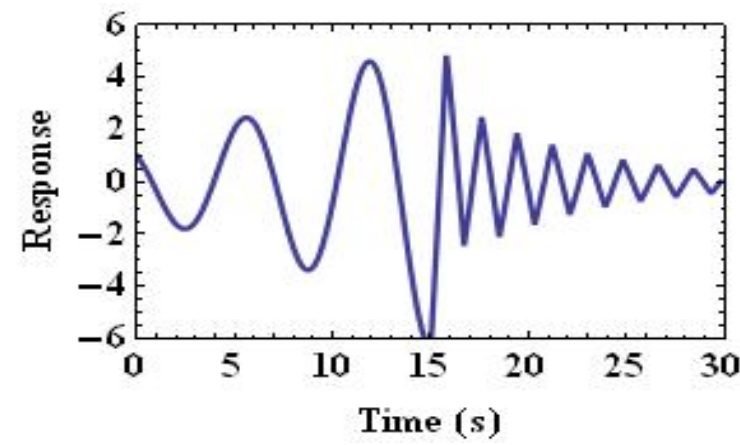

consequent control are interrupted the system becomes

Figure 2: Evolution of simple hybrid system

unstable. Pulsed illumination by an electromagnetic wave can cause such an interruption but must continue for substantial time compared to system operating times.

\section{SCADA SYSTEM}

The Directed Energy Technology Office uses a large open air test facility to test the effects of electromagnetic waves on complex systems. The building has a number of electronic systems installed to provide realistic testing of those systems [9]. Figure 3 shows a photograph of one of the SCADA

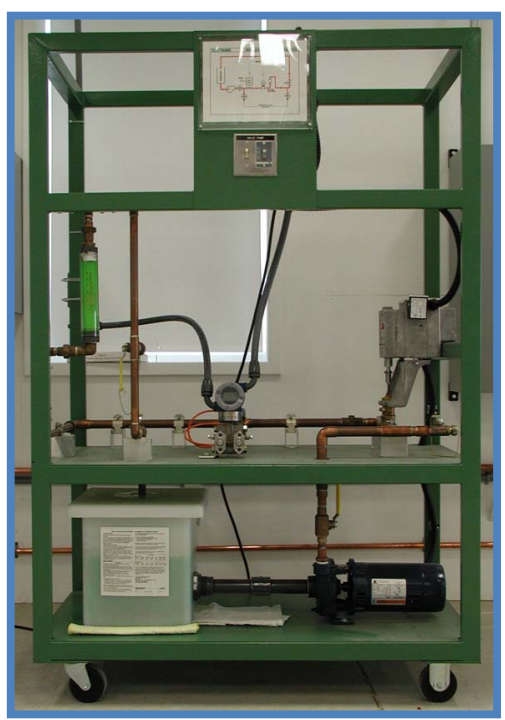

Figure 3: SCADA at NSWC test facility

systems used.

There are two SCADA system trainers. One of those systems has a tank with a level controller. The level of the tank is controlled by the pump and the release valve for the drain as shown in Fig. 4. This layout is a combination of the two test facility SCADAs.

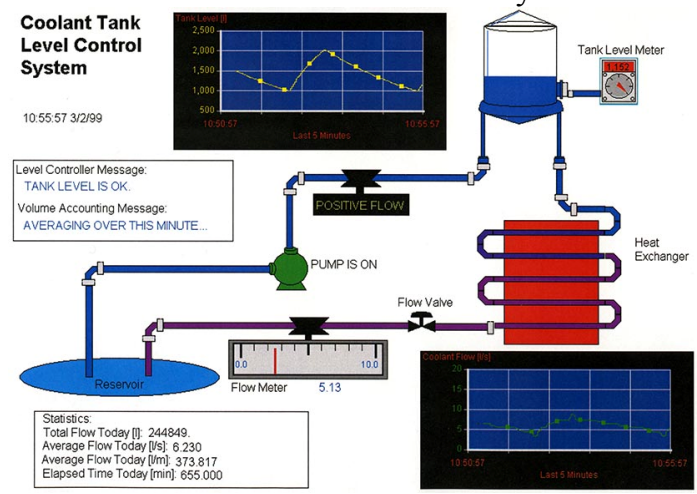

Figure 4: Level control SCADA trainer

The tank has a level sensor that is used to control the level in the tank. There is a large reservoir that forms a supply for the pump and reservoir for the drain. Since the flows in and out of the reservoir do not interact, the system is open. 


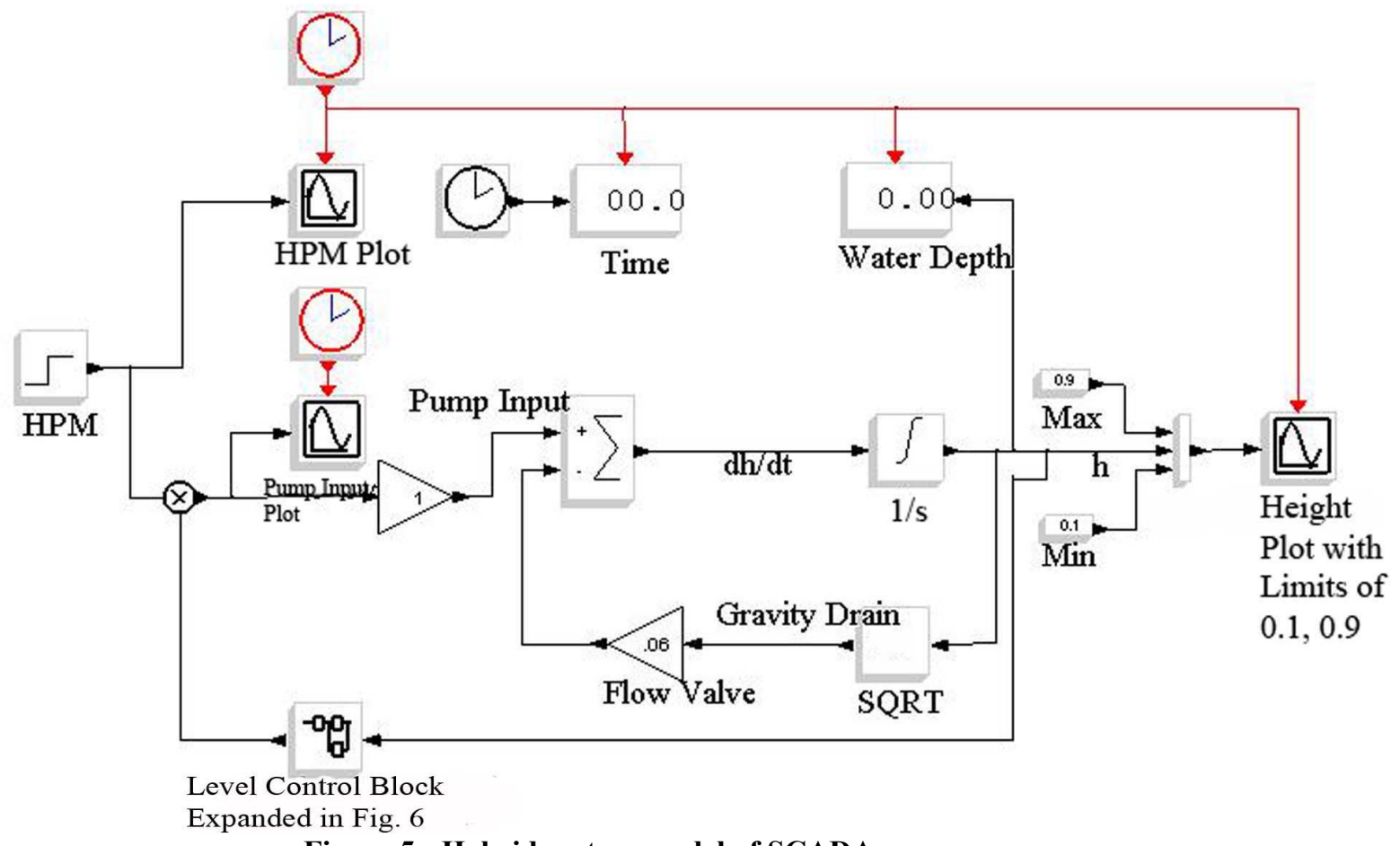

Figure 5: Hybrid system model of SCADA

\section{HYBRID SCADA MODEL}

We have developed a functional model of the SCADA system [9] in Fig. 4 using the SCICOS code [10] and that model is shown in Fig. 5. The model integrates the flows in and out of the tank to calculate the height $\mathrm{h}$ of the liquid in the tank that ranges from 0 to 1 . The liquid height is determined from integration of the pump input rate and the loss rate through the flow valve and gravity drain. Each of these systems is representative of those in system shown in Fig. 4. Rate of gravity drain is proportional to the square root of the liquid height, as is the pressure in the bottom of the tank. The level controller, shown in Fig. 6 and as a block in Fig. 5, is

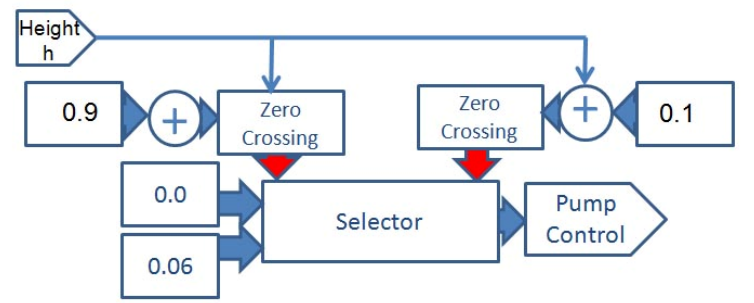

Figure 6: Level controller (expansion of block in Fig. 5)

used to maintain the level between 0.1 and 0.9 . The level controller detects a zero crossing of the rising or falling liquid as it passes the lower or upper bounds of the level and turns the pump on or off. Minus to plus and plus to minus detectors compare the height (input 1) to upper and lower limits. A selector box then chooses to turn the pump on or off depending on the depth limits. The dual state of the pump represents the hybrid part of the system.
As part of the DETO test program, current injection tests were performed on a SCADA similar to that in Figs. 4 and 5. These tests showed that the pump could be turned off by an injected current. The box labeled as HPM is just a unit step that turns off the pump at 30 seconds into the simulation consistent with the test results.

Fig. 7 shows the evolution of the liquid height for this case. The simulation starts at a level of 0.5 and drains until the control system detects a level of 0.1 . At that point, the controller starts the pump and the level rises. At 30 seconds, the pump is turned off by the simulated injected current and begins to drain again. This time, however, the pump is locked off by the injected current and the controller signal to turn the pump on is ignored - thus causing the tank to empty and system to fail.

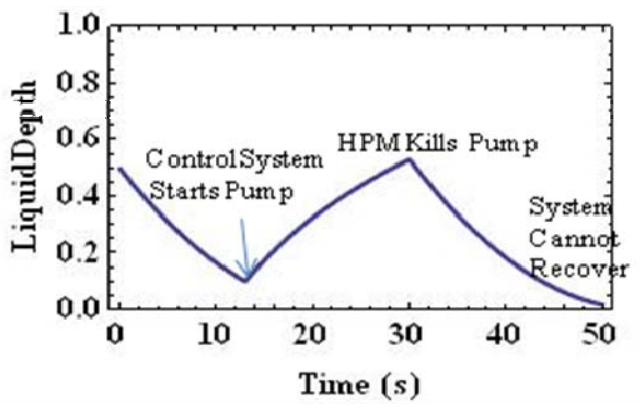

Figure 7: Evolution of hybrid model of SCADA

This model is relatively simple but its purpose is to show the development of a tool that can show functional failures resulting from many-pulse or continuous-wave illumination. In the future, applications with complex failure modes can be demonstrated. These types of control system models represent many coupled nonlinear differential 
equations. Their solution can demonstrate many failure modes including chaos.

\section{STABILITY}

The designers of all real systems expect that the systems they design will be stable when all of the control systems operate. Our purpose is to determine how a repeated "nudge" at a system resonance can bring a system down. This type of failure occurred in an oil pipe line whose pump added to the pressure in the pipe at the peak pressure point of pressure waves traveling through the pipe - eventually causing the pressure waves to increase beyond the capacity of the pipe.

Stability of complex nonlinear hybrid systems is difficult to determine [11]. Usually, the approach to showing stability is to find a bounding function that does not grow at late times (Lyapunov function). For hybrid systems, overall bounding functions are difficult to find and one must make do with bounding functions for individual states. When insult is included in the model, whose purpose is to destabilize the system, establishing stability becomes even more difficult.

\section{CONCLUSIONS}

We have described two types of models that can be used to determine the response of a complex electronic system to extended electromagnetic illumination. Our hybrid model was used to predict functional failure of a SCADA trainer and the result determined to be consistent with test data.

The hybrid model is a building block of what will be much more complex models with extremely complex failure modes. Many units of the water tank models controlled by a SCADA might be combined to represent a city-water system, for example, or a faster-responding electronic or hydraulic system.

\section{References}

[1] J. LoVetri, A. TM. Wilbers, A.P.M. Zwamborn. "Microwave Interaction with a Personal Computer: Experiment and Modeling", Proc. of 13th Int. Zurich Symposium on EMC. Zurich, Switzerland, 1999, p.203-206.

[2] R. L. Gardner and D. C. Stoudt, "Review of Susceptibility Data and Analysis Techniques in High-Power Electromagnetics", Proceedings of the USNC/URSI Meeting, Boulder, CO, Jan 2009.

[3] I. Kohlberg, R. L. Gardner, R. Boling and C. Ropiak, "Theoretical Considerations Regarding Assessment of HEMP and IEMI Upset of Digital Systems", Proceedings of the $28^{\text {th }}$ General Assembly of the International Union of Radio Science, Delhi, India, October 2005.
[4] I. Kohlberg and R. L. Gardner, "Functional and Communications Theory Models in Susceptibility Analysis", Proceedings of the North American Radio Science Meeting, Columbus $\mathrm{OH}$, June 2003.

[5] H. Y. D. Yang, "Analysis of RF Radiation Interference on Wireless Communication Systems", IEEE Ant and Wireless Prop. Ltrs, 2, 2003.

[6] A. Weinberg, "The Impact of Pulsed RFI on the Coded BER Performance of the Nonlinear Satellite Communications Channel", IEEE Trans. Comm, COM-19, 5, May 1981.

[7] I. Kohlberg, R. L. Gardner, and C. Ropiak, "Techniques Relevant to the Analysis of Electromagnetic Interference Effects on Electronic Systems", Proceedings of the International Conference on Electromagnetics in Advanced Applications, Turin, September 2005.

[8] I. Kohlberg and R. L. Gardner, "A Perspective of Electromagnetic Susceptibility of Discrete Event Dynamic Systems and Hybrid Systems", Proceedings of the European Electromagnetics Symposium, Lausanne, Switzerland, Jul. 2008.

[9] Stoudt, D. C., "Building the Capability to Perform Electromagnetics Effects Tests on Complex Systems", Proceedings of the European Electromagnetics Symposium, Lausanne, Switzerland, Jul 2008.

[10] S. L. Campbell, J. P. Chancellor and R. Nikoukhah, Modeling and Simulation in Scilab/Scicos, Springer, New York 2006.

[11] W. M. Haddad and V. Chellaboina, Nonlinear Dynamical Systems and Control, Princeton University Press, Princeton, NJ, 2008. 